\title{
REVISIÓN BIBLIOGRÁFICA DE LAS TRIGONIAS DE LIMA
}

\section{RESUMEN}

Rafaela Novoa Farro ${ }^{1,2}$

Las trigonias son bivalvos marinos fósiles, pertenecientes a la Familia Trigoniidae, la cual posee 87 especies mencionadas en el Perú, y sólo cuatro de ellas han sido identificadas en la Región de Lima. La presente revisión bibliográfica tiene como finalidad incentivar a más investigadores y estudiantes a que actualicen y complementen las investigaciones sobre las Trigonias, ya que en el Perú no se ha desarrollado un potencial paleobiológico y bioestratigráfico; desde Rivera \& Alleman, 1974.

Palabras claves: Paleobiología, Trigonias, Región Lima.

\section{SUMMARY}

The trigonias are marine bivalves fossils, belonging to the Family: Trigoniidae, which has 87 species mentioned in Peru, and only four of them have been identified in the Region of Lima. This literature review aims to encourage more researchers and students to update and complement the studies on trigonias because in Peru has not developed paleobiological and bioestratigraphic potencial since Rivera \& Alleman, 1974.

Key words: Paleobiology, Trigonias, Region of Lima.

\section{INTRODUCCIÓN}

Las Trigonias son invertebrados marinos, bivalvos no sifonados, del orden de los Trigonoidea. La mayoría de los géneros se extinguieron a finales del Cretácico y se caracterizaron por poseer una extraordinaria ornamentación.

Alrededor de 87 especies de Trigoniidae están mencionadas en el Perú, dentro de las cuales algunas figuran con nombres sinónimos, otras están ubicadas en géneros no actualizados y algunas especies, descritas al inicio del siglo por C. Lisson, necesitan una descripción complementaria debido al avance de los conocimientos taxonómicos a nivel de la Familia.

Se han encontrado 4 únicas especies de Trigonias pertenecientes al Cretáceo de Lima, ubicadas en la Formación La Herradura, en los Miembros: La Virgen y La Herradura; y en la Isla San Lorenzo.

Actualmente existe un único género vivo: Neotrigonia (Sur de Australia.).

Lamentablemente no contamos con antecedentes para el presente trabajo, ya que no se han realizado trabajos anteriores sobre este tema, sólo contamos con el apoyo bibliográfico de los autores que han hecho investigaciones y descubrimientos sobre las Trigonias, razón por la cual se justifica el presente trabajo y cuyo objetivo principal, es realizar una documentación actualizada sobre las Trigonias.

La presente investigación se realizó con la recopilación de información de publicaciones originales de autores como Dana, Lisson, Alleman \& Rivera; además de búsquedas bibliográficas apersonadas en los museos de la URP y UNI, de la observación de fósiles tipos de las colecciones de las mismas universidades y de breves referencias de Internet.

\section{ASPECTOS GEOLÓGICOS DE LA REGIÓN LIMA}

Estructuralmente, Lima pertenece al Sistema de Los Andes Centrales del Perú, sin embargo por los tipos de sedimentos que se distribuyen en la franja costera, se diferencian dos zonas geográficas. A estas dos zonas se les denominará: "Región Lima” y "Cretáceo de Lima”. Región Lima.

Su principal característica es el de no aflorar el piso Albiano. Sus límites son: Hacia el Norte, la Ciudad de Ancón, hacia el Sur la Ciudad de Chilca, hacia el Este las

Facultad de Ciencias Biológicas, Universidad Ricardo Palma - Museo de Historia Natural "Vera Alleman H." Sección de Paleontología. Av. Benavides 5440, Santiago de Surco. Lima 33- Perú. E-mail: rafaelanovoafarro@gmail.com

Trabajo de investigación premiado por la Sociedad Geológica del Perú - 2009 
estribaciones Andinas y hacia el Oeste el Océano Pacífico (incluyendo los sedimentos de la Isla San Lorenzo).

\section{Cretáceo de Lima.}

Denominado así al área que comprende la "Región de Lima" más los terrenos aflorantes, ya sea al Norte o Sur de ella. Ésta área fue estudiada paleontológicamente por Lisson (1938), siendo su principal característica el de presentar afloramiento del Albiano.

Todas las Trigonias presentes en la Región Lima, pertenecen a la edad del Valanginiano. (Cuadro 1)

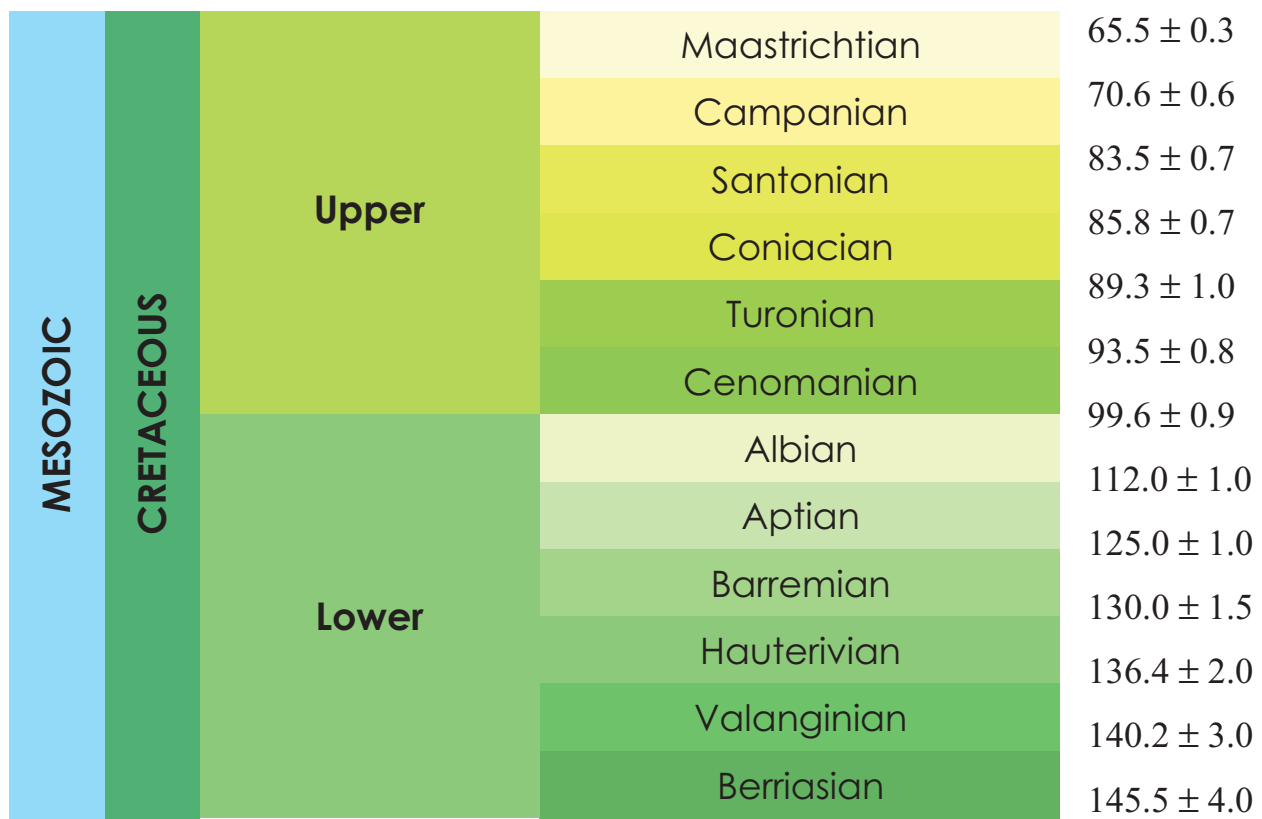

Cuadro 1. Ubicación Estratigráfica, (Geologic Time Scale 2008)

\title{
ASPECTOS PALEONTOLÓGICOS
}

\author{
SISTEMÁTICA (según Moore, 1969) \\ Clase: Bivalvia Linnè, 1758 (Buonanni, 1851) \\ Subclase: Paleoheterodonta Newell, 1965 \\ Orden: Trigonoidea Dall, 1889 \\ Superfamilia: Trigoniacea Lamarck, 1819
}

Familia: Trigoniidae Lamarck, 1819

\section{FAUNAASOCIADA: (ALLEMAN, V.1982)}

Ammonites: Raimondiceras raimondii (Gabb, 1877), Raimondiceras pfluckeri (Lisson, 1904), Argentiniceras pardoi (Lisson, 1907), Flavrella lorensis ( Lisson, 1907).

Favrella lorensis (Lisson, 1907), Raimondiceras pfluckeri (Lisson, 1904), Restos vegetales (Podozamites), Escamas de peces. (Alleman, 1985)

Bivalvos: Syrotrigonia paradisensis ( Lisson,1930), "Trigonia" lorentii (Dana, 1849), Trigonia angustecostata (Behrendsen), Panopea cf. P.carteroni (dÖrbigny), Pholadomya marcavillensis (Rivera, 1947), Cucullaea cf. C. gabrielis, Eriphyla cf. E. Avobata. (Rivera 1978).

La presencia de los ammonites Raimondiceras raimondii (Gabb, 1877) y Lissonia riveroi (Lisson), se consideran como indicadores del Valanginiano inferior y Mediano (Wiedmann, 1980).

\section{DESCRIPCIÓN DE LAS TRIGONIAS PRESEN- TES EN LAREGIÓN LIMA}

Las 4 especies de Trigonias presentes en la Región de Lima: S. paradisensis, T. ef. anguste costata, " $T$ ". lorentii y $V$. peterseni, todas han sido encontradas en dos localidades de Lima: El Morro Solar (Chorrillos) y La Isla de San Lorenzo (Callao).

- Syrotrigonia paradisensis (Isla San Lorenzo)

- T. ef. anguste costata (Isla San Lorenzo)

- "T". lorentii (Isla San Lorenzo)

- Virgotrigonia peterseni (Morro Solar)

Las cuatro especies se distinguen por su forma y ornamentación del flanco, y por la presencia de elementos concéntricos y radiales en el área. 


\section{ESPECIES}

Syrotrigonia paradisensis Lisson, 1907

Concha transversa, alargada, oblicua. Umbos delanteros, área estrecha atravesada por estrías finas. Las costillas que le siguen a las primeras, forman un codo y después se convierten en un nudo, dividiéndolas así en semicostillas marginales y semicostillas anteriores. El interespacio de las semicostillas marginales es mucho menor que el de las semicostillas anteriores.

\section{Trigonia ef. anguste- costata Berhendsen}

Umbo agudo y curvo. Carena marginal con escotaduras apretadas poco desarrolladas. Corselete estriado radialmente. El escudo no se señala distintamente: parece la prolongación del corselete. El intervalo entre las últimas costillas no alcanza a un milímetro.

\section{“Trigonia” lorentii Dana, 1850}

Concha sub-triangular, alargada. Umbos delanteros cortos, no salientes. Parte principal cubierta por dos series de costillas cruzadas, pero que no se atraviesan. Las costillas traseras arrancan cerca de la carena marginal de un modo exabrupto y son anchas, cortas, con un curso ligeramente curvo. Escudo estrecho y alargado.

Virgotrigonia peterseni Alleman, 1985

Concha alargada, fuertemente inequilateral. Umbo prominente y anterior. Escudete liso. Área angosta, llevando costillas concéntricas en la cercanía del umbo. El flanco presenta un surco antecarenal; su ornamentación consiste en dos sistemas de costas espaciadas que se unen en una posición relativamente anterior del flanco y que confluyen hacia una orientación ortogonal.
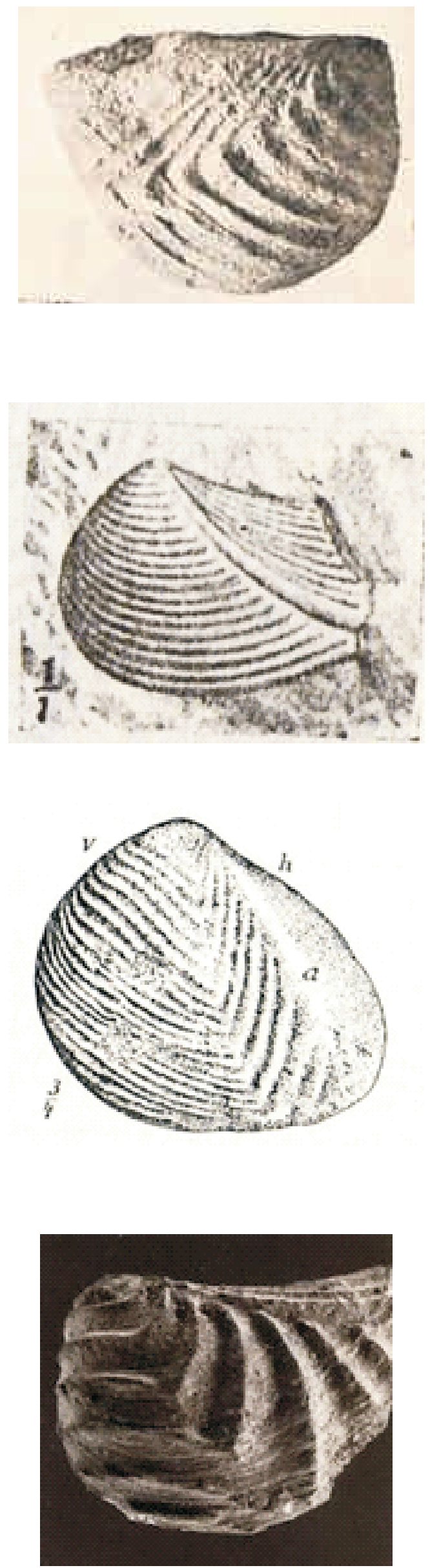


\section{CONCLUSIONES}

- Las trigonias son excelentes fósiles indicadores de calidad del hábitat y de edades.

- La única Trigonia ubicada en su unidad estratigráfica es la Virgotrigonia, que se encuentra en el Miembro La Virgen, de la Formación "La Herradura" - Morro Solar; las otras 3 están mencionadas sin que sepamos a que unidad estratigráfica pertenecen, por lo tanto seria una investigación en el futuro ubicarlos y completar este dato faltante, que es tan importante para las correlaciones geológicas.

- Se necesita recolectar material suplementario y actualizar las descripciones de las especies “ $T$ ". lorentii y $T$. anguste costata.

- Se pretende incorporar las localidades fosilíferas de las Trigonias presentes en el Cretáceo de Lima, dentro del Patrimonio Paleontológico peruano ya que cuentan con una gama de características de importancia evolutiva y paleontológica, ya mencionadas.

- El presente artículo está dirigido principalmente a estudiantes e investigadores relacionados con el área de la Biología y Geología, además del público en general, ya que se busca integrar mayor conocimiento y perfección en el tema en cuestión.

\section{AGRADECIMIENTOS}

La realización del presente trabajo ha sido posible gracias al auspicio de la Universidad Ricardo Palma y al apoyo incondicional de la Dra. Vera Alleman, profesora del área de Paleontología de la Facultad de Ciencias Biológicas de la Universidad Ricardo Palma, fundadora de la misma casa de estudios y miembro activo de la Sociedad Geológica del Perú. Un agradecimiento especial a la Sociedad Geológica del Perú por brindarme la oportunidad de participar y ser premiada en el Concurso de Monografías de Paleontología a Nivel Nacional Federico SeminarioSeminario y por el apoyo incondicional a jóvenes estudiantes que quieren incursionar en el área de Paleontología.

\section{LITERATURA CITADA}

ALLEMAN, V. 1982 Bibliografía de los Lamelibranquios fósiles del Perú y de sus Localidades con Ubicación en las Bibliotecas de Lima. Inf. Biblioteca Soc. geol. Perú (1): 68 - 82.

ALLEMAN, V. 1982. Fósiles de la Región de Lima. Revista No 5, Universidad Ricardo Palma: 118127.

ALLEMAN, V. 1985 Investigaciones sobre Trigoniidae Peruanos. Bol. Fac. C. Biol., Universidad Ricardo Palma (1): 26-30

ALLEMAN, V. 1985 Virgotrigonia Género nuevo de Trigoniidae Lamarck, 1819 (Mollusca, Bivalvia). Bol. Soc. Geol. Perú 75: 1-8. Lams. I-II

ALLEMAN, V. 1998. Ejemplares "Tipos" de Fósiles animales de la Colección V. Alleman (partim: RPT1-RPT23). Biotempo 3. Universidad Ricardo Palma : 63-67

ALLEMAN, V. 2005 Fósiles de la Isla de San Lorenzo, Callao. 2005 Revista de Ciencias, II. Departamento Académico de Ciencias. Universidad Ricardo Palma: 35-42.

LISSON, C. 1930 Trigonias neocómicas del Perú. Bol. Minas, Ind. y Constr., ser.2, 20 (1-2): 1-26

PEREZ, d`A. y REYES, R. 1994 Catálogo de Ejemplares "Tipo", conservados en la Colección paleontológica del Servicio Nacional de Geología y Minería, Chile, 1953 -1993. Bol. Del Servicio Nacional de Geología y Minería, 46 = 1- 99.

RIVERA, R. 1978 Zonas faunísticas del Cretáceo de Lima. Bol. Soc. GEOL. Perú: 62 :19-24, 101-102

WIEDMANN， J. 1980 Palaeogeographie und Stratigraphie im Grenzbereich Jura/ Kreide SûdAmerikas. Munster. Forsch. Geol. Palaeont. 51: 27-61. 